\title{
LEPROSY IN AFRICA TODAY
}

\author{
S. G. Browne, M.D., F.R.C.P., F.R.C.S., D.T.M. \\ Leprosy Service Research Unit, Uzuakoli, Eastern Region, Nigeria
}

LEPROSY constitutes one of the biggest and most complex of the many problems confronting the medical services in Africa today (Leonard Wood Memorial, Washington, 196r; WHO, 1960) and, despite the efforts of governments and ancillary agencies and the success of sulphone therapy, leprosy will continue to be a problem-medical, administrative, economic and social-for many years to come.

It is not that leprosy is a killing disease like tuberculosis or trypanosomiasis, or that it can rapidly assume epidemic proportions like yellow fever or smallpox. It is a chronic infectious granuloma affecting mainly the peripheral nerves and the skin; but this bald statement fails to indicate both the far-reaching consequences of anæsthesia and paralysis of the extremities and deeply ingrained prejudices that have for centuries regarded leprosy as a disease apart and its victims as beyond the pale. In Africa these prejudices may produce an inertia and a callousness and a fear difficult to understand and to overcome.

While there may be reasons - historical, emotional and social - for considering leprosy as a special problem calling for special measures, the tendency now (fortunately and belatedly) is to regard leprosy as part of the public health problem of transmissible disease in an environment characterized by lack of hygiene and undernutrition. It is arguable that the progressive raising of standards of hygiene may play as great a role in the control and eventual eradication of leprosy in Africa as it did in medieval Europe, with the proviso that local circumstances may render the task even more difficult and more protracted than present-day experience is discovering in the American States of Florida, Louisiana and Texas.

Fortunately, the use of a proved drug and the application of known principles do not have to await the elucidation of all that is obscure and unknown.

\section{The Problem and its Setting}

About half of all the cases of leprosy in the world are to be found in Africa, i.e. $50 \%$ of the cases in $8 \%$ of the population. Many authorities think that the total estimated incidence of five million is too conservative and should be doubled at least.
The estimates for the continent of Africa areoas follows:

North Africa (mainly the United Arab

Republic and Morocco)

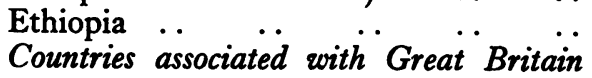

(including Nigeria, 650,000; Sierra

Leone, 100,000; Tanganyika, 100,000;

Uganda, 70,000; Ghana, 50,000;

Nyasaland, 30,000; Kenya, 25,000;

Rhodesia, 13,000; Cameroons, 6,000)

Countries associated with France (includ-

ing West Africa, 500,000; Equatorial

Africa, 150,000; Madagascar, 35,000)

Countries associated with Portugal

(Mozambique, 75,000; Angola, 30,000;

Guinea, 13,000)

Congo Republic and Ruanda Urandi

Union of South Africa ..

The accuracy of these figures varies rather wide limits: those for the ex-Belgian Congo are based on the findings of medical examinations which annually embraced two-thirds of the popelation; others, like those for Nigeria (Northefon Region), are based in part on sampling survegs and in part on detailed records covering large areas. In the main, the number of cases of leprosy under treatment is about half the estimated totals give above.

The incidence of the disease varies widely. All the larger African countries south of the Saha have a high or moderately high incidence, exceg Southern Rhodesia ( 0.7 per thousand), the Sudan ( 0.8 per thousand), Liberia (1.0 per thousand), toe Union of South Africa (I.I per thousand) and Western Nigeria (1.2 per thousand). In some parts of the continent low figures may reflect is complete data and inadequate surveys rather that the true incidence.

The highest incidences are recorded in (exFrench) Sudan (18 per thousand), Nigeria (19 pef thousand), the Congo Republic (22 per thousandy) the Central African Republic (32 per thousane) and Sierra Leone (48 per thousand). The incidence varies not only between country and country, but also within the countries and between tribe and tribe, and even between village ang village. Few districts of any size are entirely fre from leprosy; in some villages the incidence is of 
the unbelievably high order of over 500 per thousand.

The greatest concentration of cases occurs in Northern Nigeria, where there are at least 600,000 , the Congo Republic and Ethiopia.

Accurate figures are not available from all countries concerning the proportion of known cases of leprosy now under treatment. The percentages are, approximately: 5 in Sierra Leone, 20 in Ethiopia, 25 in Nyasaland and 50 in Ghana, Uganda, Northern Nigeria and the Cameroun Republic. In the ex-Belgian Congo and some of the ex-French colonies, the Union of South Africa and Eastern Nigeria the figure is over $90 \%$. The greatest numbers of untreated cases occur in Northern Nigeria, Ethiopia, Sierra Leone and Tanganyika.

Fortunately, in Africa high susceptibility is often associated with high resistance; hence there is a low lepromatous/tuberculoid ratio. In other words, the average overall incidence of 16 per thousand is offset by the comparatively mild nature of the endemic. It is broadly in those communities where cutaneous pigmentation is deepest that the proportion of tuberculoid cases is highest, varying in tropical Africa from 2 to $28 \%$, and of these a considerable proportion consists of the selflimiting, self-regressing and spontaneously healing variety. The more thorough and systematic and frequent the case-finding surveys, the greater is the proportion of such cases discovered, particularly in children and adolescents in highly endemic foci.

Extensive leprosy control schemes have not generally been in operation long enough to make a noticeable impact on country-wide incidence rates; in point of fact, the gross rates have sometimes shown apparent increases over recent years. This is due mainly to better diagnosis, to the provision of treatment and often to a changing attitude towards the disease and the possibility of cure. Elsewhere, movements of population and improved communications have led to a real increase in the incidence of leprosy and have more than offset the reduction that frequently followed urbanization and improved hygiene (including the wearing of more clothing). The lack of accurate statistics year by year is nowhere to be more regretted than in the field of leprosy.

Results in a comprehensive anti-leprosy campaign conducted for two decades through a wellorganized leprosy control service are to be seen, e.g. in the western half of Eastern Nigeria, where the incidence is now steadily declining at the rate of $15 \%$ per annum.

The attitude of the community and the individual patient to the disease varies enormously from country to country and from tribe to tribe: from a fatalistic acceptance of the will of Allah or an essentially similar fatalism in hyperendemic pagan tribes, to such a fear of the disease that its victims are driven into the bush to die, or are hidden for years by the family, who wish to conceal a disease regarded as shameful or as a mark of divine disfavour.

\section{Leprosy Control}

The provision of facilities for treatment has brought thousands of patients out of hiding.

Treatment is given to the great mass of ambulatory patients either at fixed dispensaries (rural or urban, either solely for leprosy treatment or multi-purpose), or by itinerant dispensers or teams covering regularly a circuit comprising clinics where patients from surrounding villages foregather regularly.

The segregation village, designed to harbour administratively 'open' cases (lepromatous and borderline), or those requiring para-medical supervision, have proved popular (e.g. in Nigeria, Uganda and Tanganyika) among patients, relatives and healthy neighbours, and are valuable epidemiologically.

Of the 62,000 leprosy patients at present cared for in leprosaria in Africa, about 24,000 are to be found in the countries associated with Britain (see page 86). Although the modern trend is to concentrate on ambulatory treatment in view of its? obvious financial, social and economic advantages, there is a continuing need for a central leprosarium in each endemic leprosy focus for the more adequate care of severely ill patients, for patients suffering from acute or prolonged reaction or from incipient nerve damage, and for those requiring rehabilitative surgery or physiotherapy. Mass ambulatory treatment, moreover, is not without its serious disadvantages for the individual patient who, in the course of treatment, may be susceptible to a sudden exacerbation of his clinical condition, to drug sensitivity (dermatitis, hepatitis, nephritis, acute anæmia, psychosis), to acute reaction in skin and nerves, iritis, erythema nodosum leprosum, etc.

However, given the magnitude of the problem in tropical and sub-tropical Africa, and the relatively slender resources available - financial and personal -and such factors as vast distances and poor communications and reliance on para-medical auxiliaries, mass treatment is, and will remain, the principal method of campaign.

Mass treatment, however, can make little impression on the problem of deformities and disabilities resulting from long-standing nerve involvement; such lesions are but rarely improved by oral anti-leprosy therapy. While early and adequate sulphone treatment is in general the 
surest and cheapest way to prevent such deformities, by halting the disease process before the nerves are irreversibly damaged, acute neuritis may actually be induced by drug therapy (not necessarily injudicious) in susceptible cases, and progressive fibrosis of nerve trunks (when already present) may be aggravated and accelerated by standard drug treatment.

It is estimated that $15 \%$ of the total number of leprosy patients under treatment in Africa already have deformities or disabilities of varying degree, and that perhaps $25 \%$ altogether are in need of some kind of physical rehabilitation. All forms of leprosy contribute to this incubus: the lepromatous form characterized by a widespread and progressive cutaneous granuloma, systemic deterioration and relatively late nerve involvement; the tuberculoid form, with its vigorous cellular reaction to a pauci-bacillary infection of skin and nerves, often associated with relatively early involvement of the peripheral nerve trunks in their superficial courses and consecutive trophic manifestations; and particularly the borderline forms, which are unstable and unpredictable in their neurological complications.

\section{Epidemiological Aspects}

Extensive and intensive leprosy surveys in Africa have revealed many problems.

The classical conception that prolonged and intimate contact with an 'open' case is necessary before leprosy is contracted may be generally valid, but it is belied by many recent findings. Where the incidence is low, and the population sparsely scattered, contact may be rare and speculative. Individual susceptibility (perhaps genetically inherited) and individual resistance may prove to be equally important.

It is not impossible that a single contact in a susceptible person at a propitious moment in a favourable anatomical site, when unknown conditions for transmission are optimal, may provide the portal of entry of $M$. lepra, which, after a variably long incubation period, may produce signs of overt disease.

On the analogy of skin infections, susceptibility may be inconstant and variable. If $M$. lepra is obligatorily a parasite of human tissues, as it appears to be, then contact with involved skin or with bacilliferous nasal secretions must account for new infections.

Spouses may be refractory, since the rate of conjugal infection is generally low.

While children and adolescents in a highly endemic focus may furnish a large proportion of new cases, the reason may be exposure to open cases rather than an especial susceptibility. In many surveys older people figure largely; no age is exempt, from infants in contact with one oฉ̊ two lepromatous parents to the very old.

Resistance to leprosy does not apparently depend on the general nutritional state: in someundernourished African communities there is high proportion of tuberculoid cases, while those $e^{+}$ suffering from progressive lepromatous leprosy may be among the best developed and the bes nourished individuals.

Other problems arise from the variably long incubation period of leprosy, variously termed thes ' latent' or ' silent' phase. In a given case it may be difficult or impossible to trace the source of infection.

Similarly, the absence of pain and general con $\frac{\sigma}{\delta}$ stitutional disturbance and the apparent triviality? of the early signs (e.g. vague numbness and par. æsthesiæ, skin eruptions frequently mistaken fopo atypical mycotic infections), may not only post pone treatment, but may also lead to infection of others.

\section{Clinical Aspects}

The diagnosis and differential diagnosis of leprosy in the field are predominantly clinica? problems depending on accurate observation and on a knowledge of tropical skin conditions, some of which at times may deceive the very elect. The bewildering variety of cutaneous reactions to fection with $M$. lepra is matched only by multitude of conditions that simulate it.

Moreover, the pattern of leprosy varies froms one country to another, and not only fromy continent to continent. The pattern in tropica\$ Africa differs from that in India, in Malaya, Southern Korea. The variations do not appear to̊ be directly related to race, skin pigmentation, diet climate or incidence of the disease, though ale these factors may play a part. Thus, in the Lowes Congo, peripheral nerve involvement is minima in tuberculoid leprosy and very late in the lepros: matous type, whereas it is more frequent and more serious in all types, and particularly in the border line type, in Eastern Nigeria. Again, reactiona $\$$ states may be common in one place and distinctly rare in a neighbouring district. Intermediate forms of the borderline type vary greatly i incidence. Thiocetazone (B.P.) (thiosemicarba $\overline{\bar{\pi}}$ zone) may precipitate acute reactional states in $\mathbb{Q}$ third of lepromatous patients in the Upper Congo N but not elsewhere.

A patient may present several distinct varieties of cutaneous leprosy lesions, each variety appearing at a different time, its characters depending pre sumably on little understood differences in the immunological state.

The interplay of humoral and cellular factors is leprosy is complex and obscure. The 'typica 
tuberculoid' and the 'typical leproma' are the more stable polar manifestations; in between is a confusing variety of borderline (dimorphous and intermediate) lesions, which may be highly positive or negative bacteriologically, and which may pass through phases neatly dubbed by the French poussées bacillifères. The epidemiological importance of these bacilliferous phases is evident: in the absence of known lepromatous cases in a community such patients may be responsible for the dissemination of bacilli and the perpetuation of the disease.

Another noteworthy point is the tendency of certain types of leprosy to pass through severe reactional phases spontaneously or during standard treatment. The recognition of cases liable to reaction is most important from the standpoint of therapy (a cautious build-up of drug is indicated), of neurological complications (acute and recurrent neuritis is common), of relapse (such cases require prolonged treatment) and of epidemiology (they may be highly positive bacteriologically).

In Africa the pure or primary polyneuritic type of leprosy is very rare, most apparent cases having residual tuberculoid lesions; some few, however, have polyneuritis with severe nerve pain and dramatically abrupt paralysis of the extremities, a variable period before the first skin lesion makes its appearance. Minimal skin lesions may be accompanied by widespread nerve involvement.

The persistent infectivity of the nasal mucosa after all skin smears have become negative is a little-recognized feature of leprosy in Africa. Precocious infection of the nasal mucosa is possible, but not demonstrable.

So-called ' trophic' ulceration of the extremities is very rarely a source of infection, but ulcerating lepromata of skin and nose discharge enormous numbers of acid-fast bacilli.

\section{Some Therapeutic Considerations}

Since the treatment of leprosy has been adequately reviewed recently in these columns (Jopling, 1960) the question will be but briefly referred to here.

While the sulphones undoubtedly constitute a tremendous therapeutic advance, changing the whole outlook on leprosy from administrative impotence and medical despair to hope of control and eventual eradication, optimism should be tempered by the following considerations: the average lepromatous patient requires from four to six years of treatment before clinical 'cure' with bacteriological negativity is achieved; there is a hard core of patients who, despite treatment, inexorably deteriorate and experience increasing deformity and mutilation; some lepromatous patients suffering from persistent and prolonged reaction are unable to continue treatment except under corticosteroid cover, prohibitively expensive in Africa; others remain bacteriologically positive $\triangle$ after six years of controlled treatment; clinical and bacteriological relapse is far from uncommonin a series of borderline patients the rate may be as high as $30 \%$. When these shortcomings of modern therapy are considered in conjunction with the unknown proportion of patients with tuberculoid or indeterminate leprosy whose lesions would have proved self-healing (but in whom, admittedly, resolution may be accelerated by therapy) there is little room for complacency.

While the search continues for drugs that will shorten the length of treatment and ensure more rapid sterilization of skin and nasal mucosa, there is a real need for an injectable repository sulphone that will maintain a satisfactory sulphonæmia for four weeks. Endemic areas of the ex-French colonies, for instance, are so vast and resources so slender that mobile teams cannot visit outlying groups of villages more frequently than once a month. Two leprosy research centres in Africa are investigating, on behalf of WHO, suspensions of dapsone gelled with aluminium monostearate, which give a prolonged sulphonæmia.

\section{Research}

The urgent practical problems of leprosy controf and treatment call increasingly for research into the epidemiology and pathology of the disease. Thanks to considerable work in Africa, both in the field and in institutions, there is a better understanding of many aspects of leprosy, but as long as $M$. lepra cannot be cultivated in the laboratory or inoculated successfully and reproducibly into an experimental animal many fundamental problems of infection and immunity and therapy must remain unanswered.

The four principal centres for leprosy research in Africa today are the Institut Marchoux at Bamako, in the Mali Republic; the Leprosy Service Research Unit at Uzuakoli, Eastern Nigeria; the East African Leprosy Research Centre at Busia, Tororo, Kenya; and the Westfort Leprosy Settlement, Pretoria, Union of South Africa. In addition, individual workers in Ghana Uganda, Tanganyika, the Central African Federation, Northern Nigeria and elsewhere in Africa have made and are making notable contributions to the study of leprosy. Before independence brought disruption to much of the exemplary medical services in the Belgian Congo, research was being actively prosecuted.

The four centres undertake trials of new drugs for leprosy or lepra reaction in the search for a drug that is cheap, non-toxic, suitable for mass oral or 
injectable treatment, with a prolonged action and more rapid effect than dapsone.

In addition, other aspects of leprosy are the object of research. At Bamako, research into the pattern of nerve involvement and its results is undertaken, together with the development of remedial operative procedures and rehabilitation. At Busia facilities exist for biochemical work and for following tagged drugs in the tissues. At Uzuakoli, in addition to immunological researches, a variety of investigations connected with the cutaneous and neurological manifestations of leprosy is being carried out.

Among many field enquiries may be mentioned the following: a planned BCG inoculation campaign in Northern Nigeria; epidemiological research and lepromin testing by a modified Heaf's technique in Uganda; experimental control schemes in Tanganyika; animal inoculation in the Cameroun Republic. While such activities as attempts at culturing $M$. lepra, experimental inoculation, serology and electron microscopy are best carried out in better equipped centres, routine investigation of diverse current problems in leprosy and laboratory cover of clinical trials must be undertaken in Africa itself. Research in leprosy impinges on many disciplines: clinically on dermatology and neurology, the problems of pigmentation, orthopædics, allergy, drug sensitivity, etc., and pathologically on bacteriology, immunology, histopathology, etc.

Leprosy was the first bacterial disease to be associated with a specific micro-organism; it is among the last to yield its secrets, and in the slow investigation of its numerous outstanding problems light may possibly be shed on pathological processes in other diseases.

\section{Organization and Administration}

The inspiration and success of leprosy campaigns depend largely on the knowledge, enthusiasm, initiative and resource of those in charge. Governments that have appointed leprologists and specialist staff invest wisely.

Leprosy advisory boards (representing medical, administrative and lay interests) function well in some countries.

Notwithstanding the commendable recent extension of anti-leprosy services in Africa, treatment is at present available for at the most only one out of every two persons needing it, and facilities are grossly inadequate for surgical, mental and economic rehabilitation and for occupational therapy.

Treatment of leprosy is mostly on a voluntary basis, with varying degrees of advice and persuasion-personal, communal and administrative. Voluntary nocturnal isolation is practised infrequently. Compulsory segregation has been the custom in some tribes for many years, e.g. i苗 north-east Congo and in parts of Tanganyika 3 but non-medical diagnosis may miss early lepro 2 matous leprosy and consign to the bush persons. with obvious but non-infectious lesions or wits bacteriologically negative trophic ulceration of the extremities.

Compulsory administrative segregation has $\overline{\bar{p}}$ wherever practised, usually defeated its purpos $\bar{\Phi}$ and has led to the prolonged concealment of pre cisely those patients who are a danger to theif fellows. The Union of South Africa, alone of al\$ the countries in Africa, practises obligator $\overrightarrow{\mathrm{z}}$ segregation (domiciliary or institutional) of als clinically active cases of leprosy, whether 'opent or ' closed'.

Case-finding surveys form the basis of moder leprosy campaigns, since recognition of the disease and spontaneous presentation for treatment ares both wanting in those backward rural communitieso where leprosy is most prevalent. The metho $\$$ varies: there may be teams of auxiliary workers whose primary or sole purpose is to examine an entire population for leprosy (as in the Katsin\& survey in Northern Nigeria) or leprosy detection may be incidental to a yaws mass treatment cam paign (e.g. those sponsored by WHO in Eastern. Nigeria and elsewhere); or again leprosy diagnosis may be incidental in periodical medical examina $N$ tions (as in the model FOREAMI [ref. I] and ot Peg surveys in the ex-Belgian Congo). The surves may be designed eventually to cover the whole

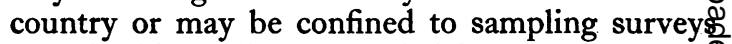
organized by a clinician-statistician team.

In all cases bacteriological control of all suspected cases of lepromatous and borderline leprosy is advisable, smears being taken from selected areas of skin (including ear lobes and the lesion) and the nasal mucosa, and stained by Ziehl-Neelsen's method by standard techniques.

When the documentation is sufficiently coms prehensive the next step is provision of facilities for treatment. Where all-purpose rural dis@ pensaries already function the addition of anti leprosy treatment is a relatively simple mattes (though often impeded by local prejudice and fear). In vast areas of Central Africa, however such facilities do not yet exist and the antip leprosy service has to be created, perhaps replacing a trypanosomiasis or yaws service now in desues tude, and eventually providing the nucleus for $\mathrm{w}$ rural health service.

The ultimate aim should be the consolidation of the anti-leprosy service and its integration into functioning dispensary service, mainly rural, an of the immediate objects are to render open caseș non-infectious, to prevent deformities, and to protect contacts. As the service develops there 
should be-thanks to mass propaganda, health education and the visual advertisement of arrested disease-early detection and regular treatment of all new infections.

The special training of medical auxiliaries in leprosy diagnosis and control is an indispensable part of any such mass campaign in Africa, and most countries have their schemes and schools.

It is to be hoped that courses in African university medical schools may not only include lecturedemonstrations on leprosy (as at Ibadan), but may be increasingly orientated towards the problems of public health and preventive medicine and so popularize services that have hitherto attracted mainly expatriate doctors in missionary or government service.

The actual system of treatment adopted depends on local circumstances, particularly on the incidence of the disease and on geographical and social factors; it also depends on the resources available in men and finance and on the political stability of the country concerned. There have been regrettable setbacks recently in more than one African country.

Where voluntary domiciliary isolation can be practised, and nocturnal isolation of lepromatous parents from susceptible offspring, valuable prophylaxis may result. In addition, although adequate supporting evidence is not yet available, prophylactic dapsone for weaned infants living with an infectious parent is recommended for as long as the patient is under treatment.

Practical problems concern continuity of treatment, the assiduity of patients, the accessibility of patients where all-season roads are nonexistent, the temptations confronting patients and auxiliaries to buy and sell dapsone on the black market; regular medical and bacteriological examinations in a sparsely populated territory.

Problems more clinical than administrative concern the criteria of ' arrest' and 'cure', the need for regular re-examination of discharged patients, and the possibilities of relapse. In general, a small proportion of typical tuberculoid cases relapses, and these relapses are not, as a rule, serious; few adequately treated lepromatous patients relapse, but cases of borderline leprosy show a relatively high tendency to relapse, as do atypical tuberculoid cases that have passed through one or more reactional phases.

The back-log of deformity and mutilations repre- sents as formidable a challenge as does the prevention of such complications in patients now undergoing treatment for leprosy. The problem is far too vast and complex to be tackled, unassisted, by poor emergent countries faced with economic difficulties and unable to devote more than ro to $12 \%$ of a restricted budget to medical services, including leprosy. One compensating feature of the problem is that most African patients requiring rehabilitation and orthopædic surgery are peasant farmers who need stability of hands and feet and freedom from ulceration rather than fine movements. Education and propaganda, ideal in theory. are beset with numerous practical difficulties limiting their applicability to Africa.

The financial and technical assistance of UNICEF and WHO is proving of enormous benefit to Africa today. Assisted projects are now being implemented in Nigeria (Eastern and Northern Regions), in the republics comprised in the ex-French territories in West and Equatorial Africa, in Ghana and in Uganda and elsewhere, and 'very satisfactory progress' has been recorded.

The Mission to Lepers, the American Leprosy Missions, the British Leprosy Relief Association and other bodies are supporting generously various projects in African countries. Missionsin many lands the pioneers in leprosy treatmentare still in the forefront, though their share of the work is proportionately declining as governments increasingly assume responsibility for all endemic disease. In schemes of rehabilitation missions are still in the van of progress. Governments subsidize co-operating missions.

\section{Future Outlook}

The extent of the problem of leprosy in Africa is approximately known and the therapeutic and administrative measures for its control are available. Over vast areas schemes and campaigns are in operation or in the planning stage.

The 'tools' are adequate, if not ideal; but ' the job' cannot be finished in an atmosphere of financial stringency or political uncertainty. The present situation constitutes a continuing challenge to governments and ancillary bodies and men of goodwill everywhere.

My thanks are due to Dr. S. E. Onwu, M.V.O., O.B.E., Director of Medical Services and Permanent Secretary, Ministry of Health, Eastern Nigeria, for permission to publish this paper.

\section{REFERENCES}

Fonds Reine Elisabeth pour l'Assistance Medicale aux Indigenes du Congo Belge: Annual Reports, Brussels. Jopling, W. H. (1960): The Treatment of Leprosy, Postgrad. med. F., 36, 634 . LEONARD WoOd MEMORIal, Washington (1961): Leprosy Briefs, 12, 9 and 10, 33.

World Health Organization (1960): Chronicle, 14, 3. 